\title{
A GEOMETRIC PROPERTY OF CERTAIN PLANE SETS
}

\author{
KENNETH PIETZ
}

Abstract. Suppose $K$ is a compact subset of the plane of the form $\overline{\Delta(0,1)} \backslash \bigcup_{n=1}^{\infty} \Delta\left(p_{n}, r_{n}\right)$ where $\overline{\Delta\left(p_{n}, r_{n}\right)} \subseteq \Delta(0,1)$ for each $n$ and $\overline{\Delta\left(p_{i}, r_{i}\right)} \cap \overline{\Delta\left(p_{j}, r_{j}\right)}=\varnothing$ for $i \neq j$. Let $\alpha=\sup _{i>1}\left(\left(r_{i}+1\right) / r_{i}\right)$ and define the sets $\partial_{*} K \equiv \partial \Delta(0,1) \cup\left[\cup_{n=1}^{\infty} \partial \Delta\left(p_{n}, r_{n}\right)\right]$ and $F(K) \equiv\left\{z \in K \backslash \partial_{*} K: z\right.$ is not a point of density of $K\}$. It is proved that if $\alpha<1$, then $\mathcal{K}^{1}[F(K)]=0$, where $\mathcal{H}^{1}$ denotes Hausdorff one-dimensional measure.

If $p$ is a point in the complex plane $\mathbf{C}$, we denote the set $\{z \in \mathbf{C}$ : $|z-p|<r\}$ by $\Delta(p, r)$. The Lebesgue area and Hausdorff one-dimensional measure of a measurable plane set $X$ will be denoted by $\mathcal{L}^{2}(X)$ and $\mathcal{H}^{1}(X)$, respectively. The notation $X \triangle Y$ will mean $(X \backslash Y) \cup(Y \backslash X)$.

The term "circular set" will mean a compact subset of $\mathbf{C}$ of the form $\overline{\Delta(0,1)} \backslash \cup_{n=1}^{\infty} \Delta\left(p_{n}, r_{n}\right)$, where $\overline{\Delta\left(p_{n}, r_{n}\right)} \subseteq \Delta(0,1)$ for all $n$ and $\overline{\Delta\left(p_{i}, r_{i}\right)} \cap \overline{\Delta\left(p_{j}, r_{j}\right)}=\varnothing$ for $i \neq j$. This includes a variety of sets considered in the studies of rational approximation and analytic capacity [6], [8].

For each circular set

$$
K=\overline{\Delta(0,1)} \backslash \bigcup_{n=1}^{\infty} \Delta\left(p_{n}, r_{n}\right)
$$

with $r_{i+1} \leqslant r_{i}$ for $i \geqslant 1$, define the type of $K$ to be the number $\alpha$ $=\sup _{i \geqslant 1}\left(\left(r_{i+1}\right) / r_{i}\right)$. It is clear that $0<\alpha \leqslant 1$. Furthermore, the set

$$
\partial_{*} K \equiv \partial \Delta(0,1) \cup\left[\bigcup_{n=1}^{\infty} \partial \Delta\left(p_{n}, r_{n}\right)\right]
$$

(where $\partial$ denotes topological boundary) is called the outer boundary of $K$. We will also use the notation $K_{0} \equiv K \backslash \partial_{*} K$.

If $X$ is any measurable subset of $\mathbf{C}$, a point $p \in \mathbf{C}$ will be called a point of density (respectively, rarefaction) of $X$ if

$$
\left.\lim _{r \rightarrow 0} \frac{\mathcal{L}^{2}[\Delta(p, r) \cap X]}{\pi r^{2}}=1 \quad \text { (respectively, } 0\right) .
$$

The purpose of this paper is to prove the following

THEOREM. Let $K$ be a circular set of type $\alpha<1$, and let $F(K)=\left\{z \in K_{0}: z\right.$ is not a point of density of $K\}$. Then $\mathcal{K}^{-1}[F(K)]=0$.

The proof requires some preliminaries.

If $\mu$ is any compactly supported, complex, regular Borel measure on $\mathbf{C}$, the

Received by the editors January 10, 1975.

AMS (MOS) subject classifications (1970). Primary 28A75, 46J10; Secondary 46G10.

(C) American Mathematical Society 1976 
Newtonian potential of $\mu$ is defined by

$$
U_{|\mu|}(z)=\int \frac{d|\mu|(\zeta)}{|\zeta-z|} .
$$

It is well known that $U_{|\mu|}$ is finite almost everywhere with respect to area [2]. For each $z \in \mathbf{C}$ such that $U_{|\mu|}(z)<\infty$, the Cauchy transform of $\mu$ is defined by

$$
\hat{\mu}(z)=\int \frac{d \mu(\zeta)}{\zeta-z} .
$$

The function $\hat{\mu}$ is thus defined $\mathfrak{L}^{2}$ almost everywhere, is analytic off the closed support of $\mu$, and vanishes at infinity.

If $f$ is a complex function with first order partial derivatives, we write

$$
\frac{\partial f}{\partial z}=\frac{1}{2}\left(\frac{\partial f}{\partial x}-i \frac{\partial f}{\partial y}\right) \text { and } \frac{\partial f}{\partial \bar{z}}=\frac{1}{2}\left(\frac{\partial f}{\partial x}+i \frac{\partial f}{\partial y}\right)
$$

where $z=x+i y$.

If $\mu$ is a measure as indicated above, it is a consequence of Green's theorem that $\partial \hat{\mu} / \partial \bar{z}=-\pi \mu$ in the sense of distributions. A discussion of this and other properties of the function $\hat{\mu}$ may be found in Chapter 3 of [2].

We have $K=\overline{\Delta(0,1)} \backslash \cup_{n=1}^{\infty} \Delta\left(p_{n}, r_{n}\right)$. Let $\mu$ be the measure on $K$ which equals $d z / 2 \pi i$ on $\partial \Delta(0,1)$ and $-d z / 2 \pi i$ on $\partial \Delta\left(p_{n}, r_{n}\right), n=1,2, \cdots$. Then $\hat{\mu}=\chi_{K} E^{2}$ almost everywhere, where $\chi_{K}$ denotes the characteristic function of $K$, and $\partial \hat{\mu} / \partial \bar{z}=-\pi \mu$. Since $\hat{\mu}$ is real, it follows that $\partial \hat{\mu} / \partial z$ is also a measure. Thus, grad $\hat{\mu}$ is a vector-valued measure, which means that the set $K$ has finite perimeter in the sense of DeGiorgi [3], [4].

With Federer [5], we say that a unit vector $u$ is an exterior normal of $K$ at $z$ if and only if

$$
\lim _{r \rightarrow 0} \frac{\mathfrak{L}^{2}[w:|w-z|<r,(w-z) \cdot u<0, w \notin K]}{r^{2}}=0
$$

and

$$
\lim _{r \rightarrow 0} \frac{\mathfrak{L}^{2}[w:|w-z|<r,(w-z) \cdot u>0, w \in K]}{r^{2}}=0
$$

where $\cdot$ denotes inner product. Such a unit vector $u$, if it exists, is uniquely determined by $K$ and $z$, and is denoted by $\nu(K, z)$. In case no such $u$ exists, $\nu(K, z)$ is the null vector. This defines, for each $z \in \mathbf{C}$, a vector $\nu(K, z)$ $=\nu_{1}(K, z)+i \nu_{2}(K, z)$.

Let $N(K)$ be the subset of $\mathbf{C}$ at which $\nu(K, z) \neq 0$, i.e., the set at which an exterior normal to $K$ exists. Since $K$ has finite perimeter, Federer's theorem [5] implies that

$$
\frac{\partial}{\partial x} \chi_{K}=\frac{\partial \hat{\mu}}{\partial x}=-2 \pi \operatorname{Re} \mu=\nu_{1}(K, z) d \mathcal{H}^{1}(z)
$$

and

$$
\frac{\partial}{\partial y} \chi_{K}=\frac{\partial \hat{\mu}}{\partial y}=-2 \pi \operatorname{Im} \mu=\nu_{2}(K, z) d \mathcal{K}^{1}(z)
$$


in the sense of distributions, where $\mu$ is the measure on $K$ defined above. Since $\mu$ is supported on $\partial_{*} K$, this implies the following lemma.

LEMma 1. The equality $\mathcal{K}^{1}\left[\partial_{*} K \triangle N(K)\right]=0$ holds.

Following Vol'pert [7], we define the essential boundary of $K$ to be the set of all points in $\mathbf{C}$ which are neither points of density nor points of rarefaction of $K$. We write $E(K)$ for the essential boundary of $K$. The following lemma follows from the theorem of Vol'pert in subsection 4 of [7].

LEMMA 2. The relation $E(K) \supseteq N(K)$ holds. Furthermore,

$$
\mathcal{H}^{1}[E(K) \backslash N(K)]=0 .
$$

Now let $s>\sqrt{1 /\left(1-\alpha^{2}\right)}$ be fixed but arbitrary. For any $p \in K_{0}$ and $r>0$ define

$$
L(p, r)=\mathcal{H}^{1}\left\{\Delta(p, r) \cap\left[\bigcup_{n=1}^{n_{k}} \partial \Delta\left(p_{n}, r_{n}\right)\right]\right\}
$$

and

$$
A(p, r)=\mathfrak{L}^{2}\left\{\Delta(p, r) \cap\left[\bigcup_{n=1}^{n_{k}} \Delta\left(p_{n}, r_{n}\right)\right]\right\}
$$

where in each case the union on the right is taken over those finitely many $\Delta\left(p_{n}, r_{n}\right)$ such that $r_{n}>r / 2 s$.

LEMmA 3. There exists a number $\beta>0$ with the following property. For all $p \in K_{0}$ and $r>0$ such that $L(p, r) \leqslant \beta r$, we have

$$
A(p, r)<\frac{\pi}{4 s^{2}}\left(s^{2}-\frac{1}{1-\alpha^{2}}\right) r^{2} .
$$

To prove the lemma, consider a fixed $p \in K_{0}$ and $r>0$. Define a function $f:[0, r) \rightarrow\left[0, \pi r^{2}\right)$ by

$$
\begin{aligned}
f(t) & =f(p, r ; t) \\
& =\sup \left\{\mathfrak{L}^{2}\left[\Delta(p, r) \cap \bigcup_{j=1}^{m} \Delta_{j}\right]: \mathcal{H}^{1}\left[\Delta(p, r) \cap \bigcup_{j=1}^{m} \partial \Delta_{j}\right] \leqslant t\right\}
\end{aligned}
$$

where the supremum is taken over all finite collections of disks $\left\{\Delta_{j}\right\}$ whose closures are pairwise disjoint and do not contain $p$. It is clear that $f^{-1}(0)$ $=\{0\}$ and that $f$ is nondecreasing and continuous at the origin. We complete the proof of the lemma by letting $\beta$ be any positive number such that

$$
f(\beta r)<\frac{\pi}{4 s^{2}}\left(s^{2}-\frac{1}{1-\alpha^{2}}\right) r^{2},
$$

and noting that the same $\beta$ works for all $r<1$, by homothetic transformation.

Returning to the proof of the main theorem, we choose $\beta$ as in Lemma 3 and define $S=\left\{p \in K_{0}\right.$ : for all sufficiently small $\left.r>0, L(p, r) \geqslant \beta r\right\}$. Since $|\mu|(S)=0$, it follows from Theorem 3 of [1] that $\mathcal{K}^{1}(S)=0$. 
Let $T=K_{0} \backslash S$ and consider an arbitrary $p \in T$. There exists a sequence $t_{k} \searrow 0$ such that $L\left(p, t_{K}\right)<\beta t_{K}$ and hence

$$
A\left(p, t_{k}\right)<\frac{\pi}{4 s^{2}}\left(s^{2}-\frac{1}{1-\alpha^{2}}\right) t_{k}^{2} \quad \text { for } k=1,2, \cdots .
$$

Let $t_{k}$ be fixed and consider those $\Delta\left(p_{n}, r_{n}\right)$ with $r_{n}<t_{k} / 2 s$ which intersect $\Delta\left(p, t_{k}\right)$. A simple computation, using the fact that $K$ is of type $\alpha$, shows that $\mathbb{L}^{2}\left[\Delta\left(p, t_{k}\right) \backslash K\right]<\pi t_{k}^{2} / 4$. It follows that $p$ is not a point of rarefaction of $K$.

The proof of the theorem is completed by summarizing the above results. Lemma 1 implies that $\mathfrak{H}^{-1}\left[K_{0} \cap N(K)\right]=0$ whence $\mathfrak{K}^{-1}\left[K_{0} \cap E(K)\right]=0$ by Lemma 2. However, $K_{0}$ is the disjoint union of $S$ and $T$, with $\mathcal{K}^{-1}(S)=0$. Since $T$ contains no points of rarefaction, we have therefore

$$
\mathfrak{K}^{-1}[F(K)]=\mathfrak{K}^{-1}[F(K) \cap T]=\mathfrak{K}^{-1}[E(K) \cap T]=0 \text {. Q.E.D. }
$$

\section{REFERENCES}

1. A. S. Besicovitch, $A$ general form of the covering principle and relative differentiation of additive functions. I, Proc. Cambridge Philos. Soc. 41(1945), 103-110. MR7, 10.

2. Andrew Browder, Introduction to function algebras, Benjamin, New York, 1969. MR39 \#731.

3. Ennio DeGiorgi, Su una teoria generale della misura $(r-1)$-dimensionale in uno spazio ad $r$ dimensioni, Ann. Mat. Pura Appl. (4) 36(1954), 191-213. MR15, 945.

4. Nuovi teoremi relativi alle misure $(r-1)$-dimensionali in uno spazio ad $r$ dimensioni, Ricerche Mat. 4(1955), 95-113. MR17, 596.

5. Herbert Federer, A note on the Gauss-Green theorem, Proc. Amer. Math. Soc. 9(1958), 447-451, MR20 \#1751.

6. T. W. Gamelin, Uniform algebras, Prentice-Hall, Englewood Cliffs, N.J., 1969.

7. A. I. Vol'pert, The spaces BV and quasilinear equations, Mat. Sb. 73(115)(1967), 255-302 = Math. USSR Sb. 2(1967), 225-267. MR35 \#7172.

8. Lawrence Zalcman, Analytic capacity and rational approximation, Lecture Notes in Math., vol. 50, Springer-Verlag, New York and Berlin, 1968. MR37 \#3018.

Lockheed Electronics Company, Inc., Aerospace Systems Division, Mail Code B-12, Houston, TeXas 77058 